\title{
The comorbidities of dysmenorrhea: a clinical survey comparing symptom profile in women with and without endometriosis
}

This article was published in the following Dove Press journal: Journal of Pain Research

\author{
Susan F Evans' \\ Tiffany A Brooks ${ }^{2}$ \\ Adrian J Esterman ${ }^{3,4}$ \\ $M$ Louise Hull ${ }^{5}$ \\ Paul E Rolan ${ }^{6}$ \\ 'School of Medicine, University of \\ Adelaide, Adelaide, SA, Australia; \\ ${ }^{2}$ School of Psychology, University \\ of South Australia, Adelaide, SA, \\ Australia; ${ }^{3}$ School of Nursing and \\ Midwifery, University of South \\ Australia, Adelaide, SA, Australia; \\ ${ }_{4}^{4}$ James Cook University, Cairns, \\ QLS, Australia; ${ }^{5}$ Robinson Research \\ Institute, School of Pediatrics and \\ Reproductive Health, University of \\ Adelaide, Adelaide, SA, Australia; \\ ${ }^{6}$ School of Medicine, University of \\ Adelaide, Adelaide, SA, Australia
}

Correspondence: Susan F Evans School of Medicine, University of Adelaide, PO Box 4025, Norwood South, Adelaide, 5067, SA, Australia

Tel +61418840895

Fax $+6|8836329| I$

Email susan.evans@adelaide.edu.au
Purpose: Dysmenorrhea is a common disorder that substantially disrupts the lives of young women. The frequency of 14 associated symptoms both within and outside the pelvis was determined.

Patients and methods: Symptom questionnaires were completed by 168 women with dysmenorrhea, allocated to three groups based on their diagnostic status for endometriosis confirmed (Endo + ), endometriosis excluded (Endo-), or endometriosis diagnosis unknown (No Lap). Those with endometriosis confirmed were further divided into current users (Endo+ $\mathrm{Hx}+$ ) and non-users of hormonal treatments (Endo $+\mathrm{Hx}-$ ). Users of hormonal treatments were further divided into users (Endo+ Hx+ LIUCD+) and non-users (Endo+ Hx+ LIUCD-) of a levonorgestrel-releasing intra-uterine contraceptive device (LIUCD). The frequency and number of symptoms within groups and the effect of previous distressing sexual events were sought.

Results: Women with and without endometriosis lesions had similar symptom profiles, with a mean of 8.5 symptoms per woman. Only $0.6 \%$ of women reported dysmenorrhea alone. The presence of stabbing pelvic pains was associated with more severe dysmenorrhea $(P=0.006)$, more days per month of dysmenorrhea $(P=0.003)$, more days per month of pelvic pain $(P=0.016)$, and a diagnosis of migraine $(P=0.054)$. The symptom profiles of the Endo+ $\mathrm{Hx}+$ and Endo+ $\mathrm{Hx}-$ groups were similar. A history of distressing sexual events was associated with an increased number of pain symptoms $(P=0.003)$.

Conclusion: Additional symptoms are common in women with dysmenorrhea, and do not correlate with the presence or absence of endometriosis lesions. Our study supports the role of central sensitization in the pain of dysmenorrhea. The presence of stabbing pelvic pains was associated with increased severity of dysmenorrhea, days per month of dysmenorrhea, days per month of pelvic pain, and a diagnosis of migraine headache. A past history of distressing sexual events is associated with an increased number of pain symptoms.

Keywords: dysmenorrhea, endometriosis, headache, bladder pain syndrome, chronic pain, pelvic pain, levonorgestrel-releasing intra-uterine device, stabbing pain

\section{Introduction}

Dysmenorrhea, the experience of painful menstruation, is common. ${ }^{1}$ A study of Australian girls aged 16-18 years showed that while 93\% experienced some pain with menstruation, $21 \%$ experienced severe pain, frequently associated with disruption of life activities and school absence. ${ }^{2}$ While dysmenorrhea may be present as a sole symptom without evidence of disease, it may also be associated with the medical condition endometriosis, or may pre-date chronic pelvic pain. ${ }^{3-6}$ Endometriosis is associated with an average of 11 hours of lost workplace productivity per week, ${ }^{7}$ and pelvic pain has 
been estimated to cost the Australian economy over US\$6 billion per year. ${ }^{8}$ Dysmenorrhea in isolation, whether as a symptom of endometriosis or when associated with chronic pelvic pain, is clearly a condition with important educational, employment, quality of life, financial, and health economic implications.

Developing effective clinical guidelines for the management of dysmenorrhea, endometriosis, and chronic pelvic pain is complicated due to their overlapping symptomatology, a lack of biomarkers, and the requirement that women undergo laparoscopic surgery to determine whether endometriosis lesions are present. ${ }^{9}$ In addition, confounding factors, potentially including a past history of sexually distressing events, may modify the pain experience..$^{10}$

While several studies have linked a laparoscopic diagnosis of endometriosis with a wide range of additional symptoms at a rate higher than the general population,,$^{9,11-15}$ few papers have investigated the frequency of these symptoms in women presenting with dysmenorrhea in clinical practice. In addition, they have not determined whether a specific symptom profile is associated with the presence of endometriosis lesions.

This study investigates the prevalence of 14 symptoms in women with dysmenorrhea who were referred to a pelvic pain and endometriosis unit with ready access to laparoscopic surgery. It compares the symptom profile of women with and without a laparoscopic diagnosis of endometriosis, and the severity of symptoms in women with and without a history of distressing sexual events. Three specific hypotheses were investigated:

1. That the prevalence of additional symptoms is high in women with dysmenorrhea,

2. That a specific symptom profile may indicate the presence of endometriosis lesions, and

3. That experience of distressing sexual events may alter the symptom profile of women with dysmenorrhea.

This study provides an insight into both the pain experience and the symptom profile of women presenting to gynecologists and laparoscopic surgeons in real-life clinical practice.

\section{Patients and methods Study design}

This is a cross-sectional analytical study.

\section{Participants}

The target population consisted of girls and women over 16 years of age, with dysmenorrhea rated as more than 3 on a
0-10 numerical scale, and with sufficient written English language skills to complete a symptom questionnaire. All participants were given an informed opportunity, to give or withhold consent for the use of their de-identified health information for research purposes, by marking one of two boxes on the questionnaire (see the section "Test methods" for details). Figure 1 shows the CONSORT flow diagram for study inclusion.

Permission to study the symptoms of those women who had indicated their consent on the questionnaire was approved by the Royal Adelaide Hospital Ethics Committee, HREC Reference No: R20170604. The study was conducted in accordance with the Declaration of Helsinki.

\section{Test methods}

The symptom questionnaire (displayed in Appendix 1) was posted to all new patients (301 women) referred to a pelvic pain and endometriosis unit, with ready access to laparoscopic surgery for the period January 1, 2015 to June 30, 2016. The questionnaire was developed by a pain specialist gynecologist (SFE) to assist with patient management. It was refined over 18 years to ensure ease of use by patients, minimize unanswered questions, and facilitate a full clinical assessment of relevant symptoms by the clinician.

The dysmenorrhea-associated symptoms investigated included stabbing pains in the pelvis, bowel symptoms, food intolerances, bladder symptoms, headaches, pain with sexual activity, vulval pain, fatigue, poor sleep, nausea, sweating, dizziness, anxiety, and low mood. The presence of anxiety or low mood was assessed using a Depression Anxiety Stress Scale-21 questionnaire. ${ }^{16}$

Additional questions recorded patient age, parity, use of hormonal treatments for menstrual symptoms, days of pain per month, duration of dysmenorrhea, and whether there was a history of distressing sexual events, including sexual assault.

Six months following the closure of study enrolment, notes for each patient were retrieved and reviewed by the principal investigator, who was blinded to patient symptoms. Each de-identified patient was allocated to groups according to whether a diagnosis of endometriosis had been confirmed (Endo+), excluded (Endo-), or could not be determined as no laparoscopy had been performed (No Lap). Endometriosis was considered confirmed when endometriotic lesions had been confidently observed during laparoscopy at any time in their life, or where endometriomas were confidently visualized on ultrasound at the time of clinical assessment. An ultrasound diagnosis of endometrioma has known high specificity for pathological confirmation at histology. ${ }^{17}$ Endometriosis was 


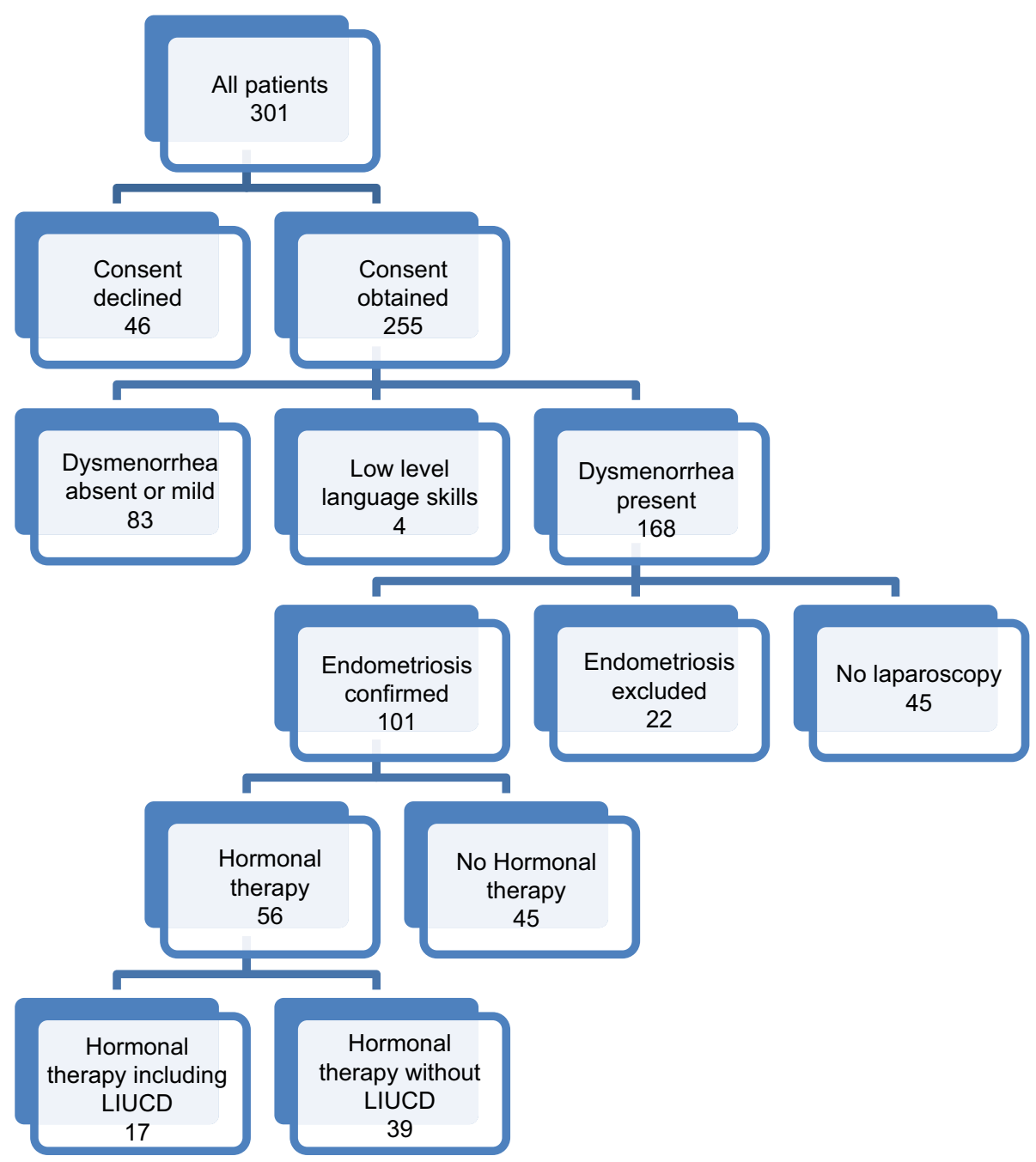

Figure I Consort diagram displaying the study selection process and method of group allocation. Abbreviation: LIUCD, a levonorgestrel-releasing intra-uterine device.

considered as excluded where at least one laparoscopy had been performed during their life, and there had never been evidence of endometriotic lesions.

\section{Statistical analysis}

Following consent, the questionnaire was de-identified, each subject was allocated a numerical code, and the de-identified data were entered into SPSS statistics 23 software (IBM Corp, Armonk, NY, USA). Questionnaire data and data analysis were undertaken by investigators blinded to endometriosis diagnosis. Statistics for each table are based on all cases with valid data in the specified range(s) for all variables.

Descriptive statistics included means and SDs for continuous variables, and counts and percentages for categorical variables. Mean and SDs were compared using independent samples $t$-tests and proportions by chi-squared tests. Predictors of stabbing pain were determined by multivariable logistic regression. Differences were considered significant at a $P$-value of $<0.05$.

\section{Missing data}

As questionnaires were completed independently, prior to clinic attendance, it was not possible to ensure that all questions were completed. Thus, for each symptom, some data were missing. The missing data rate for the 14 symptoms excluding "pain with intercourse" averaged $1.1 \%$ of responses. Missing data rate for the symptom "pain with intercourse" was $15 \%$ of responses, and missing data rate regarding history of "distressing sexual events, including sexual assault" was $18 \%$. 


\section{Results}

Details of patient numbers overall and in each group are provided in Figure 1. Of 301 women who completed the questionnaire, informed consent was provided by 255 women and declined by 25 women. Twenty-one women neglected to complete the request for consent and were deemed to have declined. Where the patient was under the age of 18 years, inclusion required the presence of a parent during the medical consultation to confirm consent.

From this cohort of 255 women, 168 patients suffered dysmenorrhea. Eighty-seven women were excluded from the study as their pain from dysmenorrhea was rated as less than 3 on a numerical $0-10$ score $(\mathrm{n}=83)$, or written English language skills were poor $(n=4)$, and consent could not reasonably be obtained.

From this cohort of 168 women, those patients with endometriosis confirmed (Endo,$+ \mathrm{n}=101$ ), endometriosis excluded (Endo-, $\mathrm{n}=22$ ), and where a diagnosis could neither be confirmed nor excluded (No Lap, $n=45$ ), were identified. Of those women with endometriosis confirmed, $16 \%$ were classified as stage 1-2 and $84 \%$ were classified as stage 3-4, using the revised American Society for Reproductive Medicine Classification. ${ }^{18}$

The cohort of 101 women with endometriosis confirmed was then divided into those patients using hormonal therapies (Endo $+\mathrm{Hx}+, \mathrm{n}=56$ ) and those patients not using hormonal therapies (Endo+ $\mathrm{Hx}^{-}, \mathrm{n}=45$ ). The hormonal therapies utilized by participants included the oral contraceptive pill $(n=33)$, a levonorgestrel-loaded intra-uterine contraceptive device (LIUCD, $n=16$ ), an oral contraceptive pill with LIUCD in combination $(n=3)$, an etonorgestrel implant $(n=6)$, depot medroxyprogesterone acetate $(\mathrm{n}=1)$, oral norethisterone $(n=2)$, and oral estradiol $(n=1)$.

From the cohort of 56 women with endometriosis confirmed who were using hormonal therapies, those women currently using hormonal treatments were further divided into those women whose hormonal therapy included a LIUCD (Endo+ Hx+ LIUCD+, n=17), and those women on hormonal therapy that did not include a LIUCD (Endo+ Hx+ LIUCD-, n=39).

Results were collated for eight patient groupings:

1. All women with dysmenorrhea (All Dys, $n=168$ )

2. Women with endometriosis confirmed (Endo,$+ n=101)$

3. Women with endometriosis excluded (Endo-, $\mathrm{n}=22$ )

4. Women with endometriosis confirmed who were current users of hormonal therapy (Endo $+\mathrm{Hx}+, \mathrm{n}=56$ )

5. Women with endometriosis confirmed who were nonusers of hormonal therapy (Endo+ $\mathrm{Hx}-, \mathrm{n}=45$ )
6. Women with endometriosis confirmed who were using hormonal therapy that included a LIUCD (Endo + Hx+ LIUCD+, $\mathrm{n}=17$ )

7. Women with endometriosis confirmed who were using hormonal therapy without a LIUCD (Endo+ $\mathrm{Hx}+$ LIUCD-, n=39)

8. Women on whom no laparoscopy had been performed (No Lap, $\mathrm{n}=45$ )

\section{Additional symptoms present per woman with dysmenorrhea}

Figure 2 displays the number of additional symptoms experienced per woman across the whole group with dysmenorrhea. Dysmenorrhea was the sole symptom in $0.6 \%$ of women, and $2.4 \%$ of women reported that all 14 symptoms were present. The mean number of additional symptoms was 8.5.

\section{Demographic, pain, and menstrual data by endometriosis diagnosis}

Patient groups All Dys, Endo+, Endo-, and No Lap were broadly similar with regard to age, age of menarche, age when dysmenorrhea commenced, frequency of previous pregnancy, and parity (Table 1). The age of participants ranged from 13 to 50 years with a mean age of 29.2 years (SD 8.4 years). Across all women, the mean severity of dysmenorrhea was 7.9 on a $0-10$ numerical scale with an SD of 1.7 .

Compared to women without endometriosis (Endo-), women with a confirmed diagnosis of endometriosis (Endo+) showed no statistically significant differences for severity of dysmenorrhea ( 8.1 vs $8.3, P=0.528$ ), days per month of

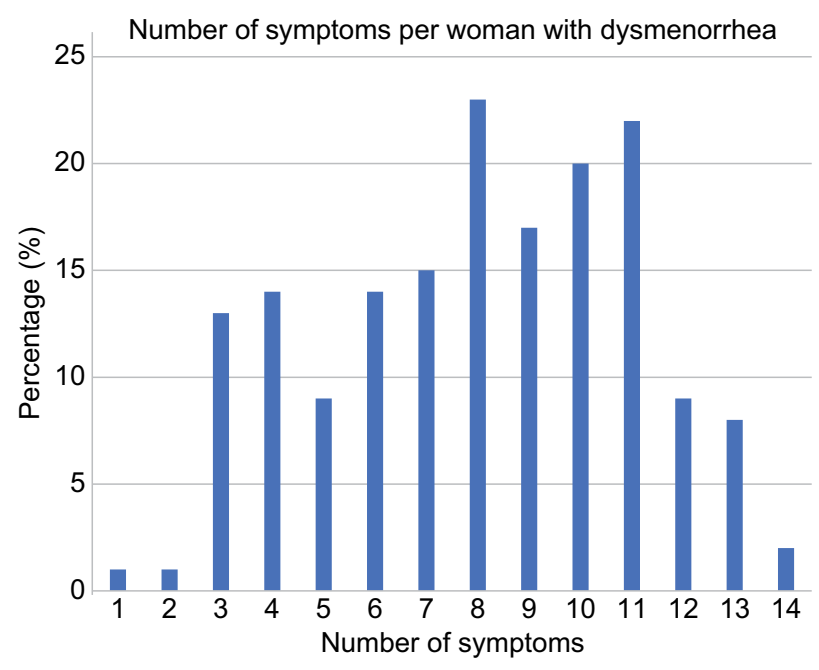

Figure 2 The percentage of women reporting between one and 14 symptoms in addition to dysmenorrhea. 
dysmenorrhea ( 8.1 vs $6.1, P=0.235$ ), days per month of pelvic pain (18.3 vs $21.1, P=0.177$ ), and parity ( $38.6 \%$ vs $45.5 \%$, $P=0.588$ ). Table 1 displays the demographic, pain, and menstrual data for each patient group by endometriosis diagnosis.

\section{Symptom profile by endometriosis diagnosis}

Table 2 displays the prevalence of each of the following symptoms across the four patient groups: stabbing pelvic pains, bowel symptoms, food intolerances, bladder symptoms, pain with sexual activity, headaches, vulval pain, fatigue, poor sleep, nausea, sweating, dizziness, anxiety, and low mood. One hundred percent of participants reported dysmenorrhea, consistent with the study inclusion criteria.
The frequency of each symptom was compared in women with endometriosis confirmed (Endo+) and women with endometriosis excluded (Endo-). The frequency of individual symptoms was similar in both groups, apart from a significantly higher frequency of bladder symptoms $(P=0.005)$ in women with endometriosis excluded. Figure 3 displays the prevalence of each symptom across the four patient groups.

\section{Demographic, pain, and menstrual data by use of hormonal therapies in women with confirmed endometriosis}

Women with confirmed endometriosis were more frequent users of a LIUCD $(15.8 \%$ vs $7.1 \%, P=0.106)$, but this difference was not statistically significant. Table 3 displays the

Table I Demographic, pain, and menstrual data by endometriosis diagnosis

\begin{tabular}{|c|c|c|c|c|c|}
\hline General demographics & $\begin{array}{l}\text { All women with } \\
\text { dysmenorrhea } \\
\text { (All Dys) } \\
(n=168)\end{array}$ & $\begin{array}{l}\text { No laparoscopy } \\
\text { (No Lap) }(n=45)\end{array}$ & $\begin{array}{l}\text { Endometriosis } \\
\text { confirmed } \\
(\text { Endo+) } \\
(n=101)\end{array}$ & $\begin{array}{l}\text { Endometriosis } \\
\text { excluded } \\
\text { (Endo-) } \\
(\mathrm{n}=22)\end{array}$ & $\begin{array}{l}\text { Significance } \\
\text { Endo+ vs } \\
\text { Endo- } \\
\text { (P-value)* }\end{array}$ \\
\hline Mean age (SD) & $29.2(8.4)$ & $29.3(8.3)$ & $29.2(8.3)$ & $28.8(9.7)$ & 0.848 \\
\hline Mean age menarche (SD) & I2.7(I.7) & $12.7(2.0)$ & $12.8(1.7)$ & $12.6(1.5)$ & 0.753 \\
\hline Previous pregnancy (\%) & 39.9 & 40 & 38.6 & 45.5 & 0.553 \\
\hline Parous (\%) & 32.7 & 31.8 & 32.0 & 38.1 & 0.588 \\
\hline \multicolumn{6}{|l|}{ Menstrual and pain data } \\
\hline Severity dysmenorrhea $0-10$ scale (SD) & $7.9(1.7)$ & $7.2(2.0)$ & $8.1(1.5)$ & $8.3(1.6)$ & 0.528 \\
\hline Age dysmenorrhea began (SD) & $16.2(6.1)$ & $16.7(6.4)$ & $15.9(5.8)$ & I6.48 (6.9) & 0.699 \\
\hline Dysmenorrhea days per month (SD) & $7.2(6.0)$ & $5.7(5.1)$ & $8.1(6.6)$ & $6.1(4.0)$ & 0.235 \\
\hline Days per month pelvic pain (SD) & $17.9(9.1)$ & $15.5(9.2)$ & $18.3(9.1)$ & $21.1(8.0)$ & 0.177 \\
\hline
\end{tabular}

Notes: All Dys, all women with dysmenorrhea, chi-squared test; Endo+, women with endometriosis confirmed; Endo-, women with endometriosis excluded; No Lap, women where no laparoscopy had been performed; *comparison of mean by independent samples $t$-test.

Table 2 Symptom prevalence by endometriosis diagnosis

\begin{tabular}{|l|l|l|l|l|l|}
\hline Symptom & $\begin{array}{l}\text { All women with } \\
\text { dysmenorrhea } \\
\text { (All Dys) (n= 168) }\end{array}$ & $\begin{array}{l}\text { No laparoscopy } \\
\text { (No Lap) (n=45) }\end{array}$ & $\begin{array}{l}\text { Endometriosis } \\
\text { confirmed (Endo+) } \\
\text { (n=10I) }\end{array}$ & $\begin{array}{l}\text { Endometriosis } \\
\text { excluded (Endo-) } \\
\text { (n=22) }\end{array}$ & $\begin{array}{l}\text { Significance } \\
\text { Endo+ vs endo- } \\
(\boldsymbol{P} \text {-value) }\end{array}$ \\
\hline Dysmenorrhea (\%) & 100 & 100 & 100 & 100 & \\
\hline Stabbing pain (\%) & 63.9 & 47.7 & 69 & 72.7 & 0.731 \\
\hline Bowel problems (\%) & 47.3 & 40.9 & 49.5 & 50.0 & 0.966 \\
\hline Food intolerances (\%) & 66.1 & 62.8 & 65 & 77.3 & 0.267 \\
\hline Bladder problems (\%) & 23.2 & 18.2 & 20 & 50 & 0.005 \\
\hline Headaches (\%) & 56 & 46.7 & 58.4 & 63.6 & 0.651 \\
\hline Sexual pain (\%) & 37.8 & 36.1 & 38.2 & 38.9 & 0.956 \\
\hline Vulval pain (\%) & 38.6 & 35.9 & 41.2 & 31.8 & 0.415 \\
\hline Fatigue (\%) & 74.4 & 68.9 & 77.2 & 72.7 & 0.652 \\
\hline Poor sleep (\%) & 57.7 & 57.8 & 58.4 & 54.5 & 0.739 \\
\hline Nausea (\%) & 49.1 & 42.9 & 48 & 66.7 & 0.12 \\
\hline Sweating (\%) & 33.9 & 28.9 & 33.7 & 45.5 & 0.296 \\
\hline Dizziness/faint (\%) & 58.9 & 48.9 & 62.4 & 63.6 & 0.912 \\
\hline Anxiety (\%) & 58.7 & 61.4 & 56.4 & 63.6 & 0.536 \\
\hline Low mood (\%) & 57.7 & 60 & 58.4 & 50 & 0.47 \\
\hline
\end{tabular}

Notes: All Dys, all women with dysmenorrhea; Endo+, women with endometriosis confirmed; Endo-, women with endometriosis excluded; No Lap, women where no laparoscopy had been performed; *chi-squared tests. 
120

Symptom profile by endometriosis diagnosis

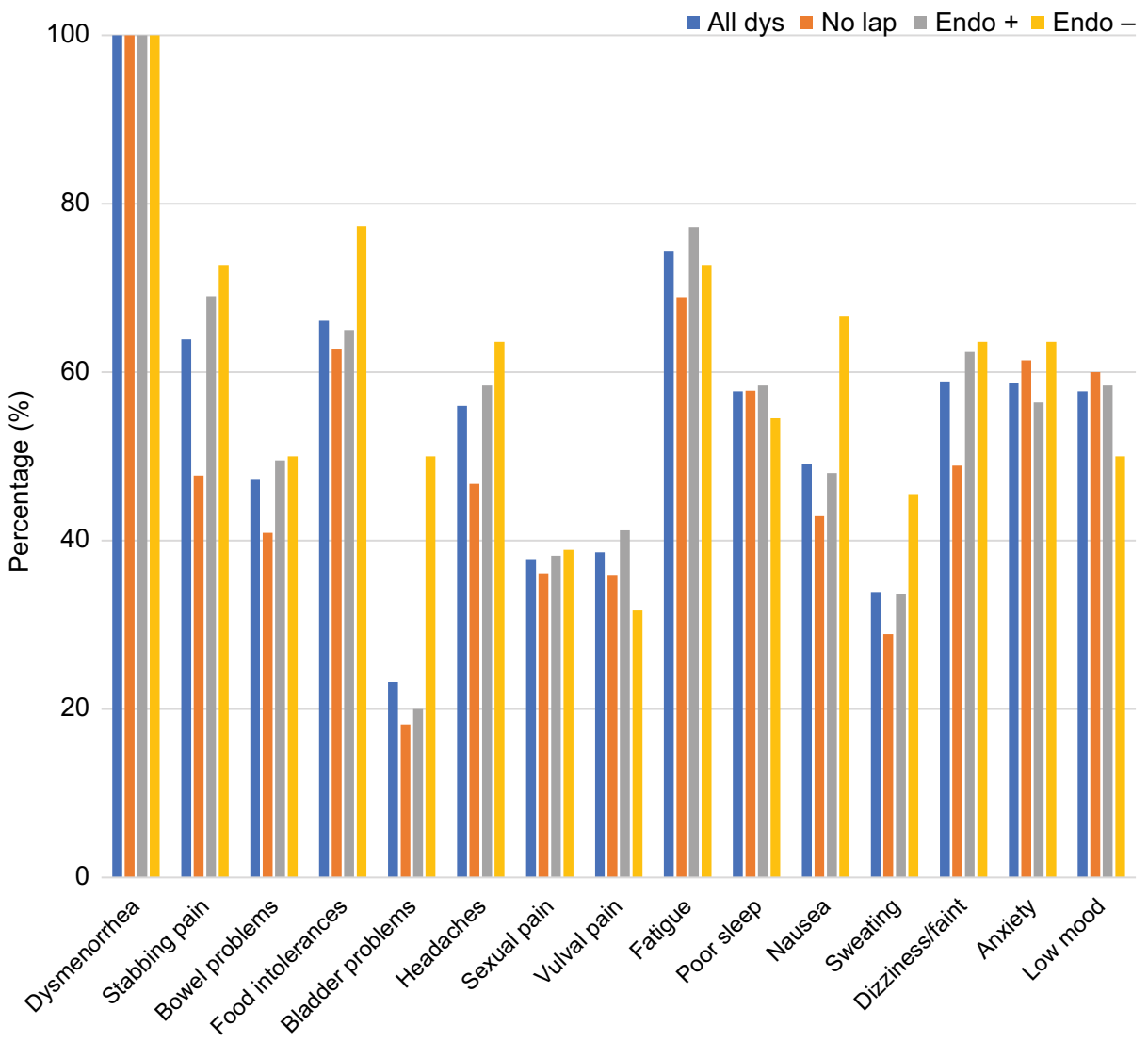

Symptoms experienced

Figure 3 The frequency of specific symptoms by endometriosis diagnosis.

Notes: All Dys, all women with dysmenorrhea; Endo+, women with endometriosis confirmed; Endo-, women with endometriosis excluded; No Lap, women where no laparoscopy had been performed.

Table 3 Demographic, pain, and menstrual data in women with endometriosis according to use or non-use of hormonal therapies and use or non-use of a levonorgestrel-releasing intra-uterine device

\begin{tabular}{|c|c|c|c|c|c|c|}
\hline General demographics & $\begin{array}{l}\text { Endo+ } \\
\mathrm{Hx}+ \\
(n=56)\end{array}$ & $\begin{array}{l}\text { Endo+ } \\
\text { Hx- } \\
(n=45)\end{array}$ & $\begin{array}{l}\text { Significance } \\
\text { Endo+ Hx+ } \\
\text { vs Endo+ Hx- } \\
\text { (P-value)* }\end{array}$ & $\begin{array}{l}\text { Endo+ } \\
\text { Hx+ } \\
\text { LIUCD+ } \\
(n=\mid 7)\end{array}$ & $\begin{array}{l}\text { Endo+ Hx+ } \\
\text { LIUCD- } \\
(n=39)\end{array}$ & $\begin{array}{l}\text { Significance } \\
\text { Endo+ Hx+ } \\
\text { LIUCD+ vs Endo+ } \\
\text { Hx+ LIUCD- } \\
(P \text {-value })^{*}\end{array}$ \\
\hline Mean age, years (SD) & $28.2(7.9)$ & $30.3(8.7)$ & 0.221 & $31.1(7.0)$ & $27.0(8.1)$ & 0.073 \\
\hline Mean age at menarche, years (SD) & $12.8(1.8)$ & $12.7(1.6)$ & 0.888 & $12.9(1.6)$ & $12.7(1.6)$ & 0.658 \\
\hline Previous pregnancy (\%) & 28.6 & 51.1 & 0.021 & 41.2 & 23.1 & 0.168 \\
\hline Parous (\%) & 22.6 & 43.2 & 0.026 & 35.3 & 16.7 & 0.13 \\
\hline \multicolumn{7}{|l|}{ Menstrual and pain data } \\
\hline Severity on dysmenorrhea $0-10$ scale (SD) & $8.3(1.5)$ & $7.8(1.4)$ & 0.167 & $8.1(1.9)$ & $8.311(1.4)$ & 0.743 \\
\hline Age dysmenorrhea began, years (SD) & $14.9(3.7)$ & $17.2(7.4)$ & 0.054 & $14.8(3.4)$ & $14.9(3.9)$ & 0.949 \\
\hline Dysmenorrhea days per month (SD) & $9.4(7.5)$ & $6.5(4.9)$ & 0.037 & $12.9(9.1)$ & $8.1(6.5)$ & 0.05 \\
\hline Pelvic pain days per month (SD) & $20.1(9.2)$ & $16.0(8.4)$ & 0.027 & $20.8(7.6)$ & $19.8(10.0)$ & $0.7 \mid 4$ \\
\hline
\end{tabular}

Notes: Endo+ $\mathrm{Hx}+$, women with endometriosis confirmed on hormonal therapy; Endo+ $\mathrm{Hx}+\mathrm{LIUCD}+$, women with endometriosis confirmed using hormonal therapy that included a LIUCD; Endo+ Hx+ LIUCD-, women with endometriosis confirmed using hormonal therapy excluding a LIUCD; Endo+ Hx-, women with endometriosis confirmed on no hormonal therapy; *comparison of mean by independent samples $t$-test, comparison of proportions by chi-squared tests.

Abbreviation: LIUCD, levonorgestrel-releasing intra-uterine contraceptive device. 
demographic, pain, and menstrual data in women with endometriosis according to use or non-use of hormonal therapies including a LIUCD.

Compared to women with endometriosis who were non-users of hormonal therapy (Endo+ $\mathrm{Hx}^{-}$), women with endometriosis who were current users of hormonal therapies (Endo $+\mathrm{Hx}+$ ) reported significantly more days of dysmenorrhea per month $(P=0.037)$, more days of pelvic pain per month $(P=0.027)$, were more likely to have had a previous pregnancy $(P=0.021)$, and were more likely to be parous $(P=0.026)$.

Compared to women with endometriosis using hormonal therapies that excluded a LIUCD (Endo+ Hx+ LIUCD-), women with endometriosis using hormonal therapies that included a LIUCD (Endo+ Hx+ LIUCD+) reported significantly more days of dysmenorrhea per month $(P=0.05)$.

\section{Symptom profile by use of hormonal therapies in women with confirmed endometriosis}

A comparison was undertaken between women with endometriosis who used (Endo+ $\mathrm{Hx}+$ ) or did not use (Endo+ $\mathrm{Hx}^{-}$) hormonal therapy, to determine if hormonal treatment was associated with a change in symptoms (Table 4). The frequency of individual symptoms was similar in both groups apart from an increase in sweating among those not receiving hormonal therapy $(P=0.009)$. Figure 4 displays the symptom profile in women with endometriosis by use of hormonal therapy.

A further comparison was undertaken between women with endometriosis using hormonal therapy that included a LIUCD (Endo+ Hx+ LIUCD+), and women with endometriosis using hormonal therapy without a LIUCD (Endo+ Hx+ LIUCD-). Those women using a LIUCD trended to a higher frequency of stabbing pains in the pelvis $(82.4 \%$ vs $63.2 \%$, $P=0.155)$ and sweating $(58.8 \%$ vs $38.5 \%, P=0.159)$ than those women using hormonal therapies without a LIUCD, but these differences were not statistically significant.

\section{Dysmenorrhea and stabbing pelvic pains}

One hundred and six of the 166 women $(63.9 \%)$ who answered the question about stabbing pains in the pelvic region reported that stabbing pains were present. Based on univariate logistic regression, variables associated with stabbing pain included: severity of dysmenorrhea $(\mathrm{OR}=1.60$, $95 \% \mathrm{CI}=1.27-2.01, P<0.001)$, number of additional symptoms $(\mathrm{OR}=1.26,95 \% \mathrm{CI}=1.12-1.41, P<0.001)$, diagnosis of migraine $(\mathrm{OR}=2.30,95 \% \mathrm{CI}=0.97-5.46, P=0.058)$, number of days per month of dysmenorrhea $(\mathrm{OR}=1.15,95 \%$ $\mathrm{CI}=1.04-1.28, P=0.007)$, and number of days per month

Table 4 Symptom prevalence across women with endometriosis, according to use or non-use of hormonal therapies, and use or nonuse of a levonorgestrel-releasing intra-uterine device

\begin{tabular}{|l|l|l|l|l|l|l|}
\hline Symptom & $\begin{array}{l}\text { Endo+ Hx+ } \\
(\mathbf{n = 5 6 )}\end{array}$ & $\begin{array}{l}\text { Endo+ Hx- } \\
(\mathbf{n = 4 5 )}\end{array}$ & $\begin{array}{l}\text { Endo+ Hx+ } \\
\text { LlUCD+ } \\
\text { (n=17) }\end{array}$ & $\begin{array}{l}\text { Endo+ Hx+ } \\
\text { LIUCD- } \\
\text { (n=39) }\end{array}$ & $\begin{array}{l}\text { Significance } \\
\text { Endo+ Hx+ } \\
\text { vs } \\
\text { Endo+ Hx- } \\
\text { (P-value)* }\end{array}$ & $\begin{array}{l}\text { Significance } \\
\text { Endo+ Hx+ LIUCD+ } \\
\text { vs } \\
\text { Endo+ Hx+ LIUCD- } \\
\text { (P-value) }\end{array}$ \\
\hline Dysmenorrhea (\%) & 100 & 100 & 100 & 100 & 1 & 1 \\
\hline Stabbing pain (\%) & 69.1 & 68.9 & 82.4 & 63.2 & 0.983 & 0.155 \\
Bowel problems (\%) & 48.9 & 50 & 47.1 & 51.3 & 0.912 & 0.771 \\
\hline Food intolerances (\%) & 66.7 & 63.6 & 52.9 & 68.4 & 0.752 & 0.270 \\
\hline Bladder problems (\%) & 20.0 & 20.0 & 17.6 & 21.1 & 1.000 & 0.770 \\
\hline Headaches (\%) & 55.6 & 60.7 & 64.7 & 59 & 0.601 & 0.686 \\
\hline Sexual pain (\%) & 35.9 & 40 & 37.5 & 41.2 & 0.693 & 0.804 \\
\hline Vulval pain (\%) & 38.6 & 43.3 & 35.3 & 47.2 & 0.635 & 0.413 \\
\hline Fatigue (\%) & 68.9 & 83.9 & 88.2 & 82.1 & 0.073 & 0.562 \\
\hline Poor sleep (\%) & 55.6 & 60.7 & 70.6 & 56.4 & 0.601 & 0.318 \\
\hline Nausea (\%) & 50 & 46.4 & 58.8 & 41 & 0.723 & 0.219 \\
\hline Sweating (\%) & 20 & 44.6 & 58.8 & 38.5 & 0.009 & 0.159 \\
\hline Dizziness/faint (\%) & 62.2 & 62.5 & 52.9 & 66.7 & 0.977 & 0.329 \\
\hline Anxiety (\%) & 57.8 & 55.4 & 52.9 & 56.4 & 0.807 & 0.810 \\
\hline Low mood (\%) & 57.8 & 58.9 & 64.7 & 56.4 & 0.907 & 0.562 \\
\hline
\end{tabular}

Notes: Endo+ $\mathrm{Hx}+$, women with endometriosis confirmed on hormonal therapy; Endo+ $\mathrm{Hx}+\mathrm{LIUCD}+$, women with endometriosis confirmed using hormonal therapy that included a LIUCD; Endo+ Hx+ LIUCD-, women with endometriosis confirmed using hormonal therapy excluding a LIUCD; Endo+ Hx-, women with endometriosis confirmed on no hormonal therapy; * chi-squared tests.

Abbreviation: LIUCD, levonorgestrel-releasing intra-uterine contraceptive device. 


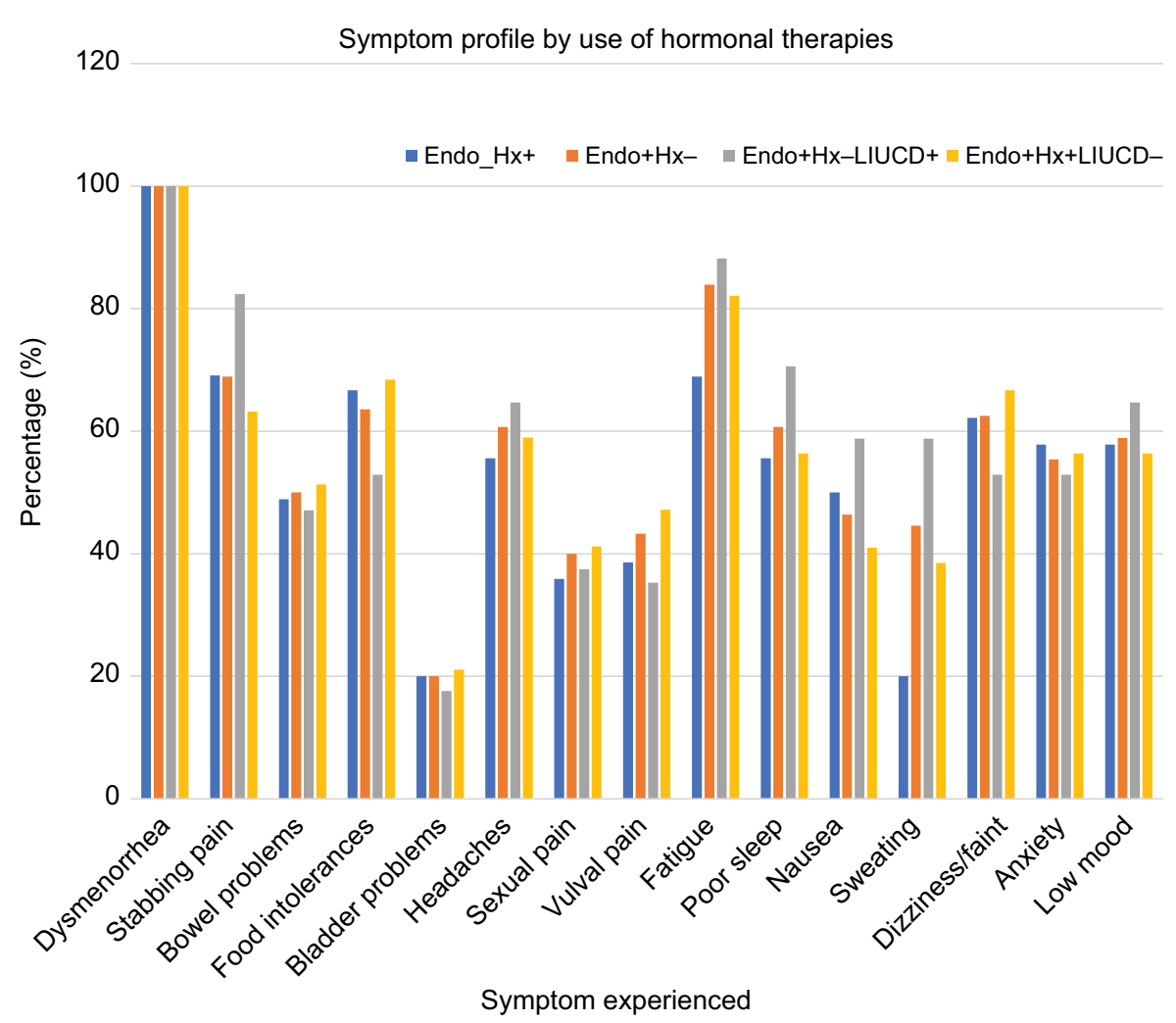

Figure 4 The frequency of specific symptoms by use or non-use of hormonal therapies, and use or non-use of a levonorgestrel-releasing intra-uterine device. Notes: Endo+ $\mathrm{Hx}+$, women with endometriosis confirmed using hormonal therapy; Endo+ $\mathrm{Hx}-$, women with endometriosis confirmed not using hormonal therapy; Endo+ $\mathrm{Hx}+\mathrm{LIUCD}+$, women with endometriosis confirmed using hormonal therapy that included a LIUCD; Endo+ Hx+ LIUCD-, women with endometriosis confirmed using hormonal therapy excluding a LIUCD.

Abbreviation: LIUCD, levonorgestrel-releasing intra-uterine contraceptive device.

of pelvic pain (OR $=1.05,95 \% \mathrm{CI}=1.01-1.08, P=0.017)$. Multivariable logistic regression found that the best joint predictors of stabbing pain were the severity of period pain ( $\mathrm{OR}=1.71,95 \% \mathrm{CI}=1.31-2.24, P<0.001)$, the number of additional symptoms (OR $=1.30,95 \% \mathrm{CI}=1.13-1.49$, $P<0.001)$, and a diagnosis of migraine ( $\mathrm{OR}=4.34,95 \% \mathrm{CI}$ $=1.46-12.87, P=0.008)$.

\section{Dysmenorrhea and headache}

One hundred and twenty-three of the 152 women (80.9\%) with dysmenorrhea who answered the question "Do you have headaches?" reported headache on at least 1 day per month. Of these, 43 women (40.2\%) reported the presence of headache on 10 or more days per month, and 26 women (21.1\%) on 15 or more days per month. The average number of days of reported headache was 10 days per month.

\section{Analysis of symptoms by past history of distressing sexual events}

Participants were asked whether they had "experienced distressing sexual events during their life, including sexual assault", with the opportunity to answer "Yes", "No", or "I prefer not to answer this question". This question was answered by $138(82.1 \%)$ of women, with $23(16.7 \%)$ answering "Yes", and 115 (83.3\%) answering "No". Figure 5 displays the dysmenorrhea score, days per month of pelvic pain, and number of symptoms in women with and without a history of distressing sexual events. Women with a history of distressing sexual events reported a significantly higher number of symptoms $(P=0.003)$ than women without a history of these events (Table 5). There was a trend toward significance for the days per month of pain (25 vs 20 days, $P=0.053)$.

\section{Discussion}

Our study has demonstrated four clinical findings. First is that additional symptoms are common in women with dysmenorrhea. Second is that the symptom profile in our study was generally independent of the presence or absence of endometriosis lesions, and independent of the use or non-use of hormonal therapy in those with confirmed endometriosis. Third is that the presence of stabbing pains in women 


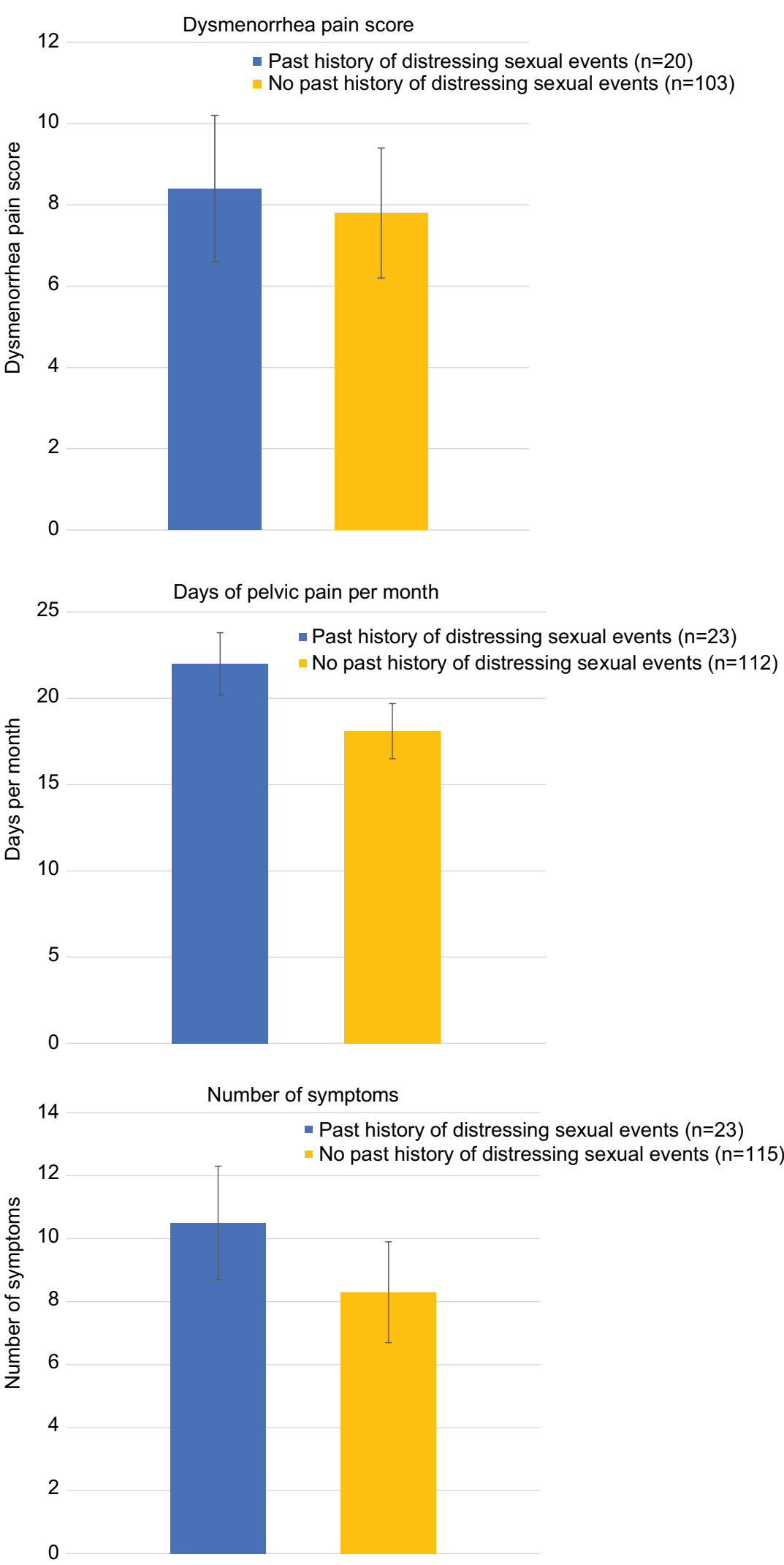

Figure 5 Dysmenorrhea pain score, days per month of pelvic pain, and number of symptoms reported in women with, and without, a history of distressing sexual events. 
Table 5 Dysmenorrhea score, days per month of pelvic pain, and number of symptoms in women with, and without, a history of distressing sexual events

\begin{tabular}{|l|l|l|l|l|l|l|l|}
\hline & \multicolumn{4}{|l|}{$\begin{array}{l}\text { Past history of distressing } \\
\text { sexual events }\end{array}$} & \multicolumn{2}{l|}{$\begin{array}{l}\text { No past history of distressing } \\
\text { sexual events }\end{array}$} & Significance* \\
\hline & N & Mean & SD & N & Mean & SD & \\
\hline Dysmenorrhea pain score & 20 & 8.4 & 1.8 & 103 & 7.8 & 1.6 & 0.112 \\
\hline Pelvic pain days per month & 23 & 22.0 & 8.4 & 112 & 18.1 & 9.0 & 0.055 \\
\hline Number of symptoms & 23 & 10.5 & 2.6 & 115 & 8.3 & 3.2 & 0.003 \\
\hline
\end{tabular}

Note: *Independent samples $t$-tests.

with endometriosis is highly associated with the severity of dysmenorrhea, days per month of dysmenorrhea, days per month of pelvic pain, and diagnosis of migraine headache. And finally that a past history of distressing sexual events is associated with an increase in the severity and number of pain symptoms.

A woman's experience of dysmenorrhea includes all symptoms experienced at menstruation, both pelvic and extra-pelvic. A major strength of this study is its symptombased applicability to the management of women presenting with dysmenorrhea and its associated symptoms, regardless of endometriosis status. While our group may represent a more complex subset of women with dysmenorrhea than is found in the general population, it is likely that they are similar to women referred to other gynecologists providing care for women with endometriosis and pelvic pain.

\section{The lack of association between pain severity, presenting symptom profile and the presence of endometriotic lesions}

Dysmenorrhea and pelvic pain have traditionally been associated with the presence of laparoscopically diagnosed endometriosis lesions. While these conditions commonly coexist, the relationship between the clinical symptoms with which a girl or woman presents to her health practitioner (dysmenorrhea) and the medical condition that may or may not be found at laparoscopy (endometriosis) remains controversial. A literature review by Janssen et $\mathrm{al}^{4}$ considered laparoscopic outcomes in adolescent girls aged 10-21 years presenting with pain. They found endometriosis lesions to be present in $62 \%$ of adolescents overall, in $75 \%$ of girls with chronic pelvic pain resistant to treatment with the oral contraceptive pill and anti-inflammatory medications, in $70 \%$ of girls with dysmenorrhea alone, and in $49 \%$ of girls with chronic pelvic pain not necessarily resistant to treatment. Additional factors affecting the experience of dysmenorrhea appear likely. A consensus paper published in 2013 included majority but not universal support for a statement describing a "philosophical shift to consideration of endometriosis and pelvic pain as a spectrum or continuum of disease". ${ }^{9}$ It was recognized that this approach would avoid excluding women who lack laparoscopic confirmation of a diagnosis of endometriosis, yet have similar symptoms and associated diagnostic and therapeutic interventions.

Robust evidence demonstrates that the presence of endometriosis lesions does not determine the severity of pelvic pain. In 656 women with chronic pelvic pain, Yosef et al found significant correlations between the severity of pain and the presence of dysmenorrhea, irritable bowel syndrome, painful bladder syndrome, pelvic muscle pain, or abdominal wall pain, but not with the presence of endometriotic lesions. ${ }^{19}$ As-Sanie et al used functional MRI to demonstrate that the experience of chronic pain was associated with anatomical changes in the brain rather than the presence of endometriosis lesions, ${ }^{20}$ and Vercellini et al confirmed the lack of association between the severity of laparoscopicallystaged endometriosis lesions and the severity of pain experienced. ${ }^{21}$ With regard to the reduction in quality of life and increase in anxiety and depression common in women with endometriosis, ${ }^{22}$ Facchin et al demonstrated that it is the presence of pain, rather than the presence of lesions, that is of importance. ${ }^{23}$ Women with asymptomatic endometriosis enjoyed the same quality of life and mental health outcomes as healthy controls. In our study, women with endometriosis excluded had a significantly higher frequency of bladder symptoms $(50 \%$ vs $20 \%, P=0.005)$ than women with endometriosis confirmed. This may reflect the presence of coexisting painful bladder syndrome, a common cause of pelvic pain that clusters with other pelvic pain diagnoses. ${ }^{24}$ By choosing to study women presenting with dysmenorrhea, rather than purely those with confirmed endometriosis, our findings further separate the pain of dysmenorrhea and the presence of endometriosis lesions. Our study also provides clinically relevant information to assist the management of women with dysmenorrhea where their endometriosis status is unknown. 


\section{The association between pain symptoms and central sensitization}

Two mechanisms consistently implicated with both the development of endometriotic lesions ${ }^{25}$ and a range of persistent visceral pain conditions ${ }^{26}$ are inflammation and activation of the innate immune system resulting in central pain sensitization. Inflammation elicits pain via inflammatory mediators and peripheral sensitization of nociceptors. ${ }^{27}$ Central sensitization may arise following continuous or recurrent nociceptive input from the periphery, resulting in enhanced excitability of spinal projection neurons in the spinal cord and central nociceptor terminals within the brain. ${ }^{28}$ Enhanced neurotransmission with long lasting molecular changes in both the spinal cord and brain may result in pain perception that no longer reflects, and is independent of, peripheral pain nociceptive signaling. ${ }^{26}$ Altered central processing of nociception in women with dysmenorrhea, both during menses and on pain-free days, as measured by serum cortisol, response to thermal stimuli, and functional MRI investigation, has been demonstrated by Vincent et al, Arendt-Nielssen et al, and Iacovides et al, ${ }^{29-31}$ with literature review by Payne et al. ${ }^{32}$ Central sensitization has the potential to affect both pelvic and extra-pelvic pain symptomatology.

With regard to pelvic symptoms, there is ample evidence for the convergence of sensory information from discrete pelvic organs of the gastrointestinal or genitourinary tract in the dorsal root ganglia, spinal cord, and brain, ${ }^{33,34}$ resulting in viscero-visceral hyperalgesia and the experience of multiple pain symptoms across pelvic organs. The women in our study demonstrated a high frequency of bowel (47.3\%) and bladder (23.2\%) symptoms. Both irritable bowel syndrome and painful bladder syndrome cluster in women with pelvic pain, and are associated with central sensitization and inflammation ${ }^{13,24,35}$

There is also evidence for the convergence of sensory information from visceral and somatic pelvic structures in the dorsal horn of the spinal cord. This results in visceral-somatic hyperalgesia and the experience of reflex muscle contraction and spasm associated with visceral pain as a manifestation of central sensitization. ${ }^{36-38}$ Our study demonstrated a high frequency of stabbing pain (63.9\%), a symptom associated with musculoskeletal pain ${ }^{39}$ in women with dysmenorrhea. The presence of stabbing pelvic pain was highly associated with the severity of dysmenorrhea, days per month of dysmenorrhea, and days per month of pelvic pain.

With regard to extra-pelvic symptoms, central pain mechanisms have been implicated in the experience of fatigue, poor sleep, fibromyalgia, and headache. ${ }^{40}$ The high frequency of chronic headache in our study is striking. About $80.9 \%$ of participants reported headache, with $21.1 \%$ reporting headaches on 15 or more days per month. In comparison, a population-based study in Italy found $42.8 \%$ of people reported headache in the previous year, and only $3.4 \%$ reported headache on 15 or more days per month..$^{41}$ These findings are consistent with growing evidence for the role of central sensitization ${ }^{20}$ and extra-pelvic symptoms in the lived pain experience of women with dysmenorrhea.

\section{Endometriosis as a common comorbidity of pelvic pain syndrome (PPS)}

Endometriosis and dysmenorrhea frequently coexist. ${ }^{4}$ However, the high frequency of symptoms in our study, both within and outside the pelvis, regardless of endometriosis status, supports the view that endometriosis and pain may be associated, rather than etiologically linked, conditions. They may share a similar underlying mechanism, yet represent distinct clinical entities within a larger syndrome of associated symptoms and conditions. Both endometriosis and dysmenorrhea would then be common, but non-essential features of female PPS, much as polycystic ovaries are common, but non-essential, features of the systemic metabolic disorder, polycystic ovarian syndrome (PCOS). ${ }^{42}$

Further support for considering endometriosis and dysmenorrhea as common features within female PPS comes from Clemens et al, who found similar pain symptoms in male PPS, where endometriosis and dysmenorrhea do not occur. ${ }^{43}$ Sutcliffe et al described similar clinical symptoms during pelvic pain flares in men and women with urologically based PPS, although flares were more frequent and of longer duration in women. ${ }^{44}$

\section{The association between pain symptoms and use of hormonal therapies including a LIUCD}

A LIUCD is a long-acting, reversible contraceptive that releases $20 \mu \mathrm{g}$ of levonorgestrel per day over 5 years of use. It offers effective contraception, reduced menstrual blood flow, reduced dysmenorrhea, and high levels of patient satisfaction in the general population, ${ }^{45,46}$ or women with adenomyosis, ${ }^{47}$ particularly in those women who have chosen to continue using a LIUCD for more than 12 months. ${ }^{48}$ However, a proportion of women request removal of the device in the first year of use, with the commonest reasons for premature device removal being pain and irregular bleed- 
ing. ${ }^{49}$ In women with endometriosis, use of a LIUCD has the added benefit of reducing the recurrence of endometriosis lesions following laparoscopic surgery. ${ }^{50}$ However, despite these known benefits of a LIUCD, Lockhat et $\mathrm{al}^{51}$ found that only $68 \%$ of women continued to use a LIUCD at 12 months post-insertion, with $11.8 \%$ of women requesting removal of the LIUCD due to "abdominal pain". Our study found that women with endometriosis using hormonal therapies reported significantly more days per month of pelvic pain, and that those using a LIUCD had a higher, but not statistically significant, increase in stabbing pains $(82.4 \%$ vs $63.2 \%, P=0.155)$ when compared with non-users of hormonal therapies. This may reflect the referral of women with pain for whom these therapies had been tried but found unsuccessful, potentially in women with more severe central pain sensitization. Research to review whether the presence of stabbing pains may indicate a subgroup of women more likely to report persistent or unresolved pain following LIUCD insertion should be considered. Our study did not include sufficient patients to investigate the relative benefits of individual oral hormonal therapies or regimes, as has been proposed in other studies. ${ }^{52,53}$

\section{The effect of previously distressing sexual events on symptom severity}

Our finding that those women who reported a history of distressing sexual events suffered more pelvic pain supports similar findings by Yosef et al. ${ }^{19}$ Their study found that a history of sexual abuse was significantly associated with increased severity of pelvic pain. Ballina et al described enhanced recovery and reduced pain severity following sexual assault in women with opioid receptor polymorphisms, further linking pain mechanisms to pain severity. ${ }^{54}$ Schliep et al found no increase in the frequency of endometriosis in women with prior history of sexual assault. ${ }^{10}$

Despite our finding that a history of distressing sexual events was associated with more severe pain, the majority of our patients reported no sexual assault. The frequency of distressing sexual events $(16.7 \%)$ is consistent with population data described in the Australian Bureau of Statistics Personal Safety Survey ${ }^{55}$ (2016), where $18 \%$ of Australian women had experienced sexual violence since the age of 15 years. With a $15 \%$ missing data rate for this question in the present study, it is possible that adverse sexual events were under-reported in our sample. However, by analyzing only those women who reported either "yes" or "no", the potential for error has been minimized.

\section{Translational significance for clinical practice}

Our study investigated 14 dysmenorrhea-associated symptoms in a group of women presenting to a gynecology clinic with particular interest in endometriosis, dysmenorrhea, and pelvic pain. This has wide-ranging clinical applications. Firstly, our study shows that the full assessment of a woman with dysmenorrhea should proceed with the expectation that multiple symptoms, both within and outside the pelvis, are likely to be present. Secondly, we found that the presenting symptom profile was an unreliable indicator of the presence of endometriosis, as similar pain profiles were found in women both with and without endometriosis lesions. Thirdly, our findings have implications for the training of health practitioners. The wide range of symptoms included within dysmenorrhea-associated PPS extends beyond the traditional teaching of individual medical and allied health care domains. There are consequently similarities between the changing management of PPS and the evolved management of PCOS. Comprehensive care of the pelvic and extra-pelvic symptoms of PCOS has moved beyond management of ovarian cysts to include the assessment of blood lipid and sugar profiles, the management of hirsuitism and acne, and lifestyle advice to manage obesity and reduce inactivity.

The high frequency of the covarying of migraine, stabbing pain, and dysmenorrhea supports further research investigating a potential common mechanism involving acquired central pain sensitization for these conditions. A pilot study by Sator-Katzenschlager et $\mathrm{al}^{56}$ using amitriptyline or gabapentin in 56 women with chronic pelvic pain found a reduction in pain among both treatment groups. If proven, this would support the use of therapeutic interventions directed at central sensitization with effects on both pelvic and extra-pelvic symptoms in women with dysmenorrhea.

\section{Conclusion}

This study aims to extend our knowledge in an area that has been under-researched with regard to its impact on the wellbeing of women, their families, and our society: the lived experience of women with dysmenorrhea.

While the lack of a control group in this study limits our ability to make firm conclusions, our findings indicate that additional symptoms are common in these women, and that the presence of endometriosis lesions cannot reliably be predicted by the presenting symptom profile. Of the symptoms investigated, the presence of stabbing pelvic pains and migraine headaches were most closely correlated with the severity of dysmenorrhea. The presence of both pelvic and 
extra-pelvic symptoms is consistent with central sensitization as a component mechanism in female PPS. A history of distressing sexual events was associated with an increased number of pain symptoms, but was not more common than in the general population.

\section{Acknowledgments}

We thank Dr N Eastaff-Leung for providing assistance in paper formatting, and Professor R Sussex for advice on editorial matters.

\section{Author contributions}

Study design, acquisition of data, preparation of data: SFE, TAB, PER, MLH. Analysis of data: SFE, AJE. Interpretation of data: SFE, PER, MLH. Preparation of article: SFE, PER, MLH. All authors contributed to data analysis, drafting and revising the article, gave final approval of the version to be published, and agree to be accountable for all aspects of the work.

\section{Disclosure}

SFE received royalties from book authorship, has received payment from Pfizer and Bayer for educational presentations, and is involved in the development of novel treatments for pelvic pain. PER is a shareholder in Havah Therapeutics and iXBiopharma, director and shareholder of Lipotek, consultant to Bionomics and Novartis, and has received payment for educational presentations from Novartis and Sequirus. The other authors report no conflicts of interest in this work.

\section{References}

1. Ju H, Jones M, Mishra G. The prevalence and risk factors of dysmenorrhea. Epidemiol Rev. 2014;36:104-113.

2. Parker MA, Sneddon AE, Arbon P. The menstrual disorder of teenagers (MDOT) study: determining typical menstrual patterns and menstrual disturbance in a large population-based study of Australian teenagers. BJOG. 2010;117(2):185-192.

3. Hardi G, Evans S, Craigie M. A possible link between dysmenorrhoea and the development of chronic pelvic pain. Aust NZJ Obstet Gynaecol. 2014;54(6):593-596.

4. Janssen EB, Rijkers AC, Hoppenbrouwers K, Meuleman C, D'Hooghe TM. Prevalence of endometriosis diagnosed by laparoscopy in adolescents with dysmenorrhea or chronic pelvic pain: a systematic review. Hum Reprod Update. 2013;19(5):570-582.

5. Jarrell J, Arendt-Nielsen L. Allodynia and Dysmenorrhea. JOGC. 2016;38(3):270-274.

6. Iacovides S, Avidon I, Baker FC. What we know about primary dysmenorrhea today: a critical review. Hum Reprod Update. 2015;21(6): $762-778$

7. Nnoaham KE, Hummelshoj L, Webster P, et al; on behalf of the World Endometriosis Research Foundation Global Study of Women's Health consortium. Impact of endometriosis on quality of life and work productivity: a multicenter study across ten countries. Fertil Steril. 2011;96(2):366-373.

8. Bush D ES Vancaillie T. The 6 Billion Woman and the \$600Million Girl: The Pelvic Pain Report. 2011. Available from: https://www.pelvicpain. org.au/pelvic-pain-report/. Accessed November 10, 2018.
9. Johnson NP, Hummelshoj L. World Endometriosis Society Montpellier Consortium. Consensus on current management of endometriosis. Hum Reprod. 2013;28(6):1552-1568.

10. Schliep KC, Mumford SL, Johnstone EB, et al. Sexual and physical abuse and gynecologic disorders. Hum Reprod. 2016;31(8):1904-1912.

11. Ulirsch JC, Ballina LE, Soward AC, et al. Pain and somatic symptoms are sequelae of sexual assault: results of a prospective longitudinal study. Eur J Pain. 2014;18(4):559-566.

12. Gyang A, Hartman M, Lamvu G. Musculoskeletal causes of chronic pelvic pain: what a gynecologist should know. Obstet Gynecol. 2013;121(3):645-650.

13. Chung MK, Chung RR, Gordon D, Jennings $C$. The evil twins of chronic pelvic pain syndrome: endometriosis and interstitial cystitis. JSLS. 2002;6(4):311-314.

14. Tietjen GE, Bushnell CD, Herial NA, Utley C, White L, Hafeez F. Endometriosis is associated with prevalence of comorbid conditions in migraine. Headache. 2007;47(7):1069-1078.

15. Maroun P, Cooper MJ, Reid GD, Keirse MJ. Relevance of gastrointestinal symptoms in endometriosis. Aust N Z J Obstet Gynaecol. 2009;49(4):411-414.

16. Lovibond PF, Lovibond SH. The structure of negative emotional states: comparison of the Depression Anxiety Stress Scales (DASS) with the Beck Depression and Anxiety Inventories. Behav Res Ther. 1995;33(3):335-343.

17. Nisenblat V, Bossuyt PM, Farquhar C, Johnson N, Hull ML. Imaging modalities for the non-invasive diagnosis of endometriosis. Cochrane Database Syst Rev. 2016;2(2):CD009591.

18. American Society for Reproductive Medicine. Revised American Society for Reproductive Medicine classification of endometriosis: 1996. Fertility and Sterility. 1997;67(5):817-821.

19. Yosef A, Allaire C, Williams C, et al. Multifactorial contributors to the severity of chronic pelvic pain in women. Am J Obstet Gynecol. 2016;215(6):760.e1-76760.

20. As-Sanie S, Harris RE, Napadow V, et al. Changes in regional gray matter volume in women with chronic pelvic pain: a voxel-based morphometry study. Pain. 2012;153(5):1006-1014.

21. Vercellini PTL, De Giorgi O, Cortesi I, Parazzini F, Crosignani PG. Endometriosis and pelvic pain: relation to disease stage and localization. Fertil Steril. 1996;65(2):299-304.

22. Laganà AS, La Rosa VL, Rapisarda AMC, et al. Anxiety and depression in patients with endometriosis: impact and management challenges. Int J Womens Health. 2017;9:323-330.

23. Facchin F, Barbara G, Saita E, et al. Impact of endometriosis on quality of life and mental health: pelvic pain makes the difference. $J$ Psychosom Obstet Gynaecol. 2015;36(4):135-141.

24. Outcault S, Clemens JQ, Ryan G, Berry SH. 1507 A qualitative analysis of symptom clustering among women diagnosed with interstitial cystitis/ painful bladder syndrome, endometriosis, vulvodynia, and overactive bladder. J Urol. 2010;183(4):e580-e581.

25. Hornung D, von Wussow U. Inflammation and endometriosis. In: Giudice LC, Evers JL, Healy DL. Endometriosis: Science and Practice. Wiley-Blackwell; 2012:255-262.

26. Dodds KN, Beckett EA, Evans SF, Grace PM, Watkins LR, Hutchinson MR. Glial contributions to visceral pain: implications for disease etiology and the female predominance of persistent pain. Transl Psychiatry. 2016;6(9):e888.

27. Grace PM, Rolan PE, Hutchinson MR. Peripheral immune contributions to the maintenance of central glial activation underlying neuropathic pain. Brain Behav Immun. 2011;25(7):1322-1332.

28. Woolf CJ, Salter MW. Neuronal plasticity: increasing the gain in pain. Science. 2000;288(5472):1765-1768.

29. Vincent K, Warnaby C, Stagg CJ, Moore J, Kennedy S, Tracey I. Dysmenorrhoea is associated with central changes in otherwise healthy women. Pain. 2011;152(9):1966-1975.

30. Arendt-Nielsen L, Madsen H, Jarrell J, Gregersen H, Drewes AM. Pain evoked by distension of the uterine cervix in women with dysmenorrhea: evidence for central sensitization. Acta Obstet Gynecol Scand. 2014;93(8):741-748. 
31. Iacovides S, Avidon I, Baker FC. Women with dysmenorrhoea are hypersensitive to experimentally induced forearm ischaemia during painful menstruation and during the pain-free follicular phase. Eur J Pain. 2015;19(6):797-804.

32. Payne LA, Rapkin AJ, Seidman LC, Zeltzer LK, Tsao JC. Experimental and procedural pain responses in primary dysmenorrhea: a systematic review. J Pain Res. 2017;10:2233-2246.

33. Malykhina AP. Neural mechanisms of pelvic organ cross-sensitization. Neuroscience. 2007;149(3):660-672.

34. Moshiree B, Zhou Q, Price DD, Verne GN. Central sensitisation in visceral pain disorders. Gut. 2006;55(7):905-908.

35. Brierley SM, Linden DR. Neuroplasticity and dysfunction after gastrointestinal inflammation. Nat Rev Gastroenterol Hepatol. 2014;11(10):611-627.

36. Gokyildiz S, Beji NK, Avcibay B, Ozgunen FT. Coping strategies of women having chronic pelvic pain. International Journal of Urological Nursing. 2013;7(3):126-132.

37. Giamberardino MA. Referred muscle pain/hyperalgesia and central sensitisation. J Rehabil Med. 2003 (41 Suppl):85-88.

38. Gillett WR, Jones D. Chronic pelvic pain in women: role of the nervous system. Expert Rev Obstet Gynecol. 2009;4(2):149-163.

39. Bergström C, Persson M, Mogren I. Pregnancy-related low back pain and pelvic girdle pain approximately 14 months after pregnancy - pain status, self-rated health and family situation. BMC Pregnancy Childbirth. 2014;14(1):48.

40. Soee AB, Thomsen LL, Kreiner S, Tornoe B, Skov L. Altered pain perception in children with chronic tension-type headache: is this a sign of central sensitisation? Cephalalgia. 2013;33(7):454-462.

41. Torelli P, Abrignani G, Berzieri L, et al. Population-based pace study: headache frequency and disease perception in adult subjects with headache. Neurol Sci. 2010;31(Suppl 1):149-151.

42. Kauffman RP, Baker TE, Baker VM, Dimarino P, Castracane VD. Endocrine and metabolic differences among phenotypic expressions of polycystic ovary syndrome according to the 2003 Rotterdam consensus criteria. Am J Obstet Gynecol. 2008;198(6):670.e1-67670.

43. Clemens JQ, Mullins C, Kusek JW, et al. The MAPP research network: a novel study of urologic chronic pelvic pain syndromes. BMC Urol. 2014;14:57.

44. Sutcliffe S, Colditz GA, Goodman MS, et al. Urological chronic pelvic pain syndrome symptom flares: characterisation of the full range of flares at two sites in the Multidisciplinary Approach to the Study of Chronic Pelvic Pain (MAPP) Research Network. BJU Int. 2014;114(6):916-925.
45. Backman T. Benefit-risk assessment of the levonorgestrel intrauterine system in contraception. Drug Saf. 2004;27(15):1185-1204.

46. Caruso S, Cianci S, Vitale SG, Fava V, Cutello S, Cianci A. Sexual function and quality of life of women adopting the levonorgestrel-releasing intrauterine system (LNG-IUS $13.5 \mathrm{mg}$ ) after abortion for unintended pregnancy. Eur J Contracept Reprod Health Care. 2018;23(1): 24-31.

47. Ji F, Yang X-H, Ai Xing Zi AL, et al. Role of levonorgestrel-releasing intrauterine system in dysmenorrhea due to adenomyosis and the influence on ovarian function. Clin Exp Obstet Gynecol. 2014;41(6):677-680.

48. Bednarek PH, Jensen JT. Safety, efficacy and patient acceptability of the contraceptive and non-contraceptive uses of the LNG-IUS. Int $J$ Womens Health. 2010;1:45-58.

49. Backman T, Huhtala S, Blom T, Luoto R, Rauramo I, Koskenvuo M. Length of use and symptoms associated with premature removal of the levonorgestrel intrauterine system: a nation-wide study of 17,360 users. BJOG. 2000;107(3):335-339.

50. Viganò P, Somigliana E, Vercellini P. Levonorgestrel-releasing intrauterine system for the treatment of endometriosis: biological and clinical evidence. Womens Health. 2007;3(2):207-214.

51. Lockhat FB, Emembolu JO, Konje JC. The efficacy, side-effects and continuation rates in women with symptomatic endometriosis undergoing treatment with an intra-uterine administered progestogen (levonorgestrel): a 3 year follow-up. Hum Reprod. 2005;20(3):789-793.

52. Caruso S, Iraci M, Cianci S, Fava V, Casella E, Cianci A. Comparative, open-label prospective study on the quality of life and sexual function of women affected by endometriosis-associated pelvic pain on $2 \mathrm{mg}$ dienogest $/ 30 \mu \mathrm{g}$ ethinyl estradiol continuous or 21/7 regimen oral contraceptive. J Endocrinol Invest. 2016;39(8):923-931.

53. Caruso S, Iraci M, Cianci S, Casella E, Fava V, Cianci A. Quality of life and sexual function of women affected by endometriosis-associated pelvic pain when treated with dienogest. $J$ Endocrinol Invest. 2015;38(11):1211-1218.

54. Ballina LE, Ulirsch JC, Soward AC, et al. $\mu$-Opioid receptor gene A118G polymorphism predicts pain recovery after sexual assault. $J$ Pain. 2013;14(2):165-171.

55. Australian Bureau of Statistics. Personal Safety, Australia. 2016. Available from: http://www.abs.gov.au/ausstats/abs@.nsf/mf/4906.0. Accessed June 9, 2018.

56. Sator-Katzenschlager SM, Scharbert G, Kress HG, et al. Chronic pelvic pain treated with gabapentin and amitriptyline: a randomized controlled pilot study. Wien Klin Wochenschr. 2005;117(21-22):761-768.
Journal of Pain Research

\section{Publish your work in this journal}

The Journal of Pain Research is an international, peer reviewed, open access, online journal that welcomes laboratory and clinical findings in the fields of pain research and the prevention and management of pain. Original research, reviews, symposium reports, hypothesis formation and commentaries are all considered for publication.

\section{Dovepress}

The manuscript management system is completely online and includes a very quick and fair peer-review system, which is all easy to use. Visit http://www.dovepress.com/testimonials.php to read real quotes from published authors. 